\title{
Special issue in Computational Modeling on Biological Systems Editorial Preface
}

\begin{abstract}
V. Moliner
Departament de Química Física i Analítica; Universitat Jaume I, 12071 Castellón (Spain)

This Special Issue is centered on questions concerning the difficulties and recent advances in the computational modeling of biological systems. Despite the expression "biological system" can enroll many different phenomena, it is focused on biochemical processes related with living organisms and, more specifically, in the foundations and applications achieved by the use of the computational chemistry on enzymatic processes and functional proteins. Enzymes are biological catalysts that speed up chemical reactions making them compatible with life. Apart from this catalytic power, often these catalysts show important advantages with respect to non-natural catalysts such as their chemo-, regio- and stereoselectivity, or the ability to work under mild conditions of temperature and pressure, which makes them the best environmental friendly catalysts to speed up the rate of chemical reactions. Nevertheless, although there have been numerous studies that have provided a solid understanding about some of the key factors of these biocatalysts, the knowledge about the origin of enzymatic efficiency to catalyze chemical reactions is still not complete. Advances in this field will contribute for their application in industry but, in order to extend their applicability to different purposes, we need a deeper understanding of their way of action. In this regard, the combination of experimental techniques and computer simulations pave the way to a quicker development in the field. The more we learn about the foundations of processes governing chemical processes in living organisms (including our bodies and cells), the better position we will have to control them with the corresponding benefits in fields such as biomedicine, biotechnology, pharmacy, etc. Thus, we could say that the developments and advances on computer simulations are closely linked to the improvements in quality of live in our planet.

This special issue contains contributions from leading experts on different sub-themes of computer simulations of enzyme related problems, starting with a review by Martin
\end{abstract}


Field who describes how the theory and practice of molecular simulation have evolved since the beginning of the 1980s, when the author started his career in this field.[1] The account is of necessity brief but highlights the changes that have had significant impact on his research and mode of working, which can be extrapolated to all scientists whose job depends on the performance of computers. The items chosen by Field in this review include technical advances that have impacted the practice of molecular simulation in addition to purely scientific developments. Donald Truhlar presents the concepts underlying the application of modern transition state theory as applied to condensedphase reactions, using enzyme-catalysed reactions as the example.[2] Truhlar shows how ensemble-averaged variational transition state theory with multidimensional tunneling (VTST/MT) contributions has proved to be a powerful method for calculating rate constants for enzyme-catalyzed reactions. Undoubtedly, this is the proper theoretical framework to connect theory and experiments. The review is closed with an interesting conclusion, which is that the leading errors are usually due more to the uncertainties in the potential energy functions than in the treatment of the dynamics per se. In the review of Alexandra Vardi-Kilshtain, Neta Nitoker and Dan Major,[3] progresses in the development of multi-scale simulation methods for the calculation of nuclear quantum effects (NQE) and accurate computation of kinetic isotope effects (KIEs) are presented using examples of several enzyme systems studied during the past years in their laboratory. The authors present developments of various novel pathintegral methods and show how the use of multidimensional free energy simulations and proper tunnelling effects calculations become as essential to properly describe KIEs and mechanisms of the selected case examples. A review of another kind of phenomenon taking place in proteins that requires a quantum description is shown by Aurélien de La Lande, Natacha Gillet, Shufeng Chen, and Dennis R Salahub.[4] From this contribution, we learn on the limitations and challenges of the most common numerical simulation approaches for the investigation of electron transfer (ET) in proteins. The review deals with direct simulation algorithms of charge migration in proteins, the applicability of the Marcus theory for ET in proteins and the tunneling aspects of electron transfer. The authors recall what has been learned on the biophysics of ET within proteins thanks to the advent of atomistic simulations. Iñaki Tuñón, Damian Laage and James Hynes present a review where some thoughts on the much debated contentious issue of dynamical effects in enzyme catalysis, and more specifically on their potential role in the acceleration of the chemical step, is offered.[5] 
To address the controversy of the term 'dynamics', they return to the TST rate constant, detailing the various sources of deviations from it due to dynamics (or not). Authors show how much can be learn by comparing the chemical step of reactions in the enzyme and in solution, and the requirement of analysing dynamical effects both in the neighbourhood of the transition state and far from it, with special attention to cases when QNE are central for the reaction.

After this block of contributions devoted to fundamental aspects of enzyme catalysis, the most important applications derived from this knowledge are shown in two reviews. The first one, written by Nuno Cerqueira, Diana Gesto, Eduardo Oliveira, Diogo Santos-Martins, Natércia Brás, Sérgio Sousa, Pedro Fernandes and Maria Ramos, provides a comprehensive overview of the receptor-based virtual screening process and of its importance in the present drug discovery and development paradigm.[6] The main stages of a virtual screening campaign, including its strengths and limitations, are the subject of particular attention in their review, with special consideration to practical issues. Recent developments in the design of biocatalysts, with particular emphasis in the de novo strategy, are reviewed by Katarzyna Świderek, Iñaki Tuñón, Vicent Moliner and Juan Bertran.[7] Based on three different reactions, Kemp elimination, Diels-Alder and retro-aldolase, the different success achieved during the last years are illustrated. As a general conclusion, the authors conclude that the interplay between new and more sophisticated engineering protocols and computational methods, based on molecular dynamics simulations with multi-scale methods and fully flexible models, seems to constitute the bed rock for present and future successful design strategies.

In the last block of this special issue, reviews on two relevant specific biological systems together with some new data papers are collected. In the first one, Alexandra Carvalho, Klaudia Szeler, Konstantinos Vavitsas, Johan Åqvist and Shina Kamerlin review the contributions of computational biology to the understanding of GTP hydrolysis on the ribosome, and in small GTPases.[8] It is stressed how, despite a wealth of biochemical, structural and computational data, the way in which GTP hydrolysis is activated and regulated is still a controversial topic, and well-designed simulations can play an important role in resolving and rationalizing the experimental data. Paramount implications can be derived from advances in this topic, keeping in mind that enzymes that hydrolyze GTP control many cellular processes. Giulia Rossetti, Domenica Dibenedetto, Vania Calandrini, Alejandro Giorgetti and Paolo Carloni illustrate, with a review of recent progress performed in their lab and other groups, how 
computational methods may be of great help to understand structure/function of neurobiologically relevant $\mathrm{G}$ protein Coupled Receptors (GPCRs) and Intrinsic Disordered proteins (IDPs).[9] in silico methods are disclosed as crucial for structural predictions of these highly relevant, fascinating and challenging systems that, otherwise, their structural characterization is lacking in most cases due, most probably to their highly flexible structural nature.

The first new data contribution to this special issue comes from Paolo Piazzetta, Tiziana Marino and Nino Russo, who give new insights in the promiscuous catalytic activity of human carbonic anhydrase and the importance of enzymatic product release.[10] The restoring step of the catalytic cycle for natural and promiscuous substrates is analyzed from the calculated potential of mean force based on the steered molecular dynamics simulations. In the following paper, the catalytic mechanism of tyrosine ammonia-lyase, an enzyme used in bioengineering due to the potential applications in industry, is explored by José Pinto, António Ribeiro, Maria Ramos, Pedro Fernandes, Marirosa Toscano and Nino Russo based on multiscale and quantum mechanical cluster models.[11] Different reaction paths are studied and energy barriers of rate limiting step of the most favorable mechanism, obtained with both models, appear to be in good agreement with experiments, which gives credit to their results. Finally, Conchín Meliá, Silvia Ferrer, Vicent Moliner and Juan Bertran present a computational study on another problem with medical applications; the mechanisms of hydrolysis of two antibiotics in the active site of a mono-nuclear $\beta$-lactamase.[12] The study, based on molecular dynamics simulations with multiscale methods, explores the reaction paths and highlights the dramatic conformational changes that can take place in the cavity of the enzyme to accommodate different substrates, which would be the origin of its substrate promiscuity. This feature would be in turn, associated to the different mechanisms that $\beta$-lactamases employ to hydrolyze the different antibiotics; i.e. the catalytic promiscuity.

We think that this Special issue represents an interesting snapshot of the state of the art on computational modeling of biological processes and, in particular, in enzyme catalyzed processes and functional proteins.

\section{Acknowledgements}


I thank the authors for the contributions, the reviewers for their always insufficiently appreciated role, and the editorial staff of $\mathrm{ABB}$ and the publisher for their continous excellent cooperation.

\section{References}

[1] Martin Field. Technical Advances in Molecular Simulation Since the 1980s. ABBI-15-56

[2] Donald Truhlar. Transition state theory for enzyme kinetics. ABBI-15-106

[3] Alexandra Vardi-Kilshtain, Neta Nitoker and Dan Major. Nuclear Quantum Effects and Kinetic Isotope Effects in Enzyme Reactions. ABBI-15-48

[4] Aurélien de La Lande, Natacha Gillet, Shufeng Chen, and Dennis R Salahub. Progress and Challenges in Simulating and Understanding Electron transfer in Proteins. ABBI-15-71

[5] Iñaki Tuñón, Damian Laage and James Hynes. Are There Dynamical Effects in Enzyme Catalysis? Some Thoughts Concerning the Enzymatic Chemical Step. ABBI-15-134

[6] Nuno Cerqueira, Diana Gesto, Eduardo Oliveira, Diogo Santos-Martins, Natércia Brás, Sérgio Sousa, Pedro Fernandes and Maria Ramos. Receptor-based virtual screening protocol for drug discovery. ABBI-15-101

[7] Katarzyna Świderek, Iñaki Tuñón, Vicent Moliner and Juan Bertran. Computational strategies for the Design of New Enzymatic Functions. ABBI-14619

[8] Alexandra Carvalho, Klaudia Szeler, Konstantinos Vavitsas, Johan Åqvist and Shina Kamerlin. Modeling the Mechanisms of Biological GTP Hydrolysis. ABBI$15-26$

[9] Giulia Rossetti, Domenica Dibenedetto, Vania Calandrini, Alejandro Giorgetti and Paolo Carloni. Structural predictions of neurobiologically relevant G-protein coupled receptors and intrinsically disordered proteins. ABBI-14-661

[10] Paolo Piazzetta, Tiziana Marino and Nino Russo. Theoretical investigation on the restoring step of the carbonic anhydrase catalytic cycle for natural and promiscuous substrates. ABBI-14-662 
[11] José Pinto, António Ribeiro, Maria Ramos, Pedro Fernandes, Marirosa Toscano and Nino Russo. New insights in the Catalytic Mechanism of Tyrosine AmmoniaLyase given by QM/MM and QM cluster models. ABBI-14-650

[12] Conchín Meliá, Silvia Ferrer, Vicent Moliner and Juan Bertran. Theoretical studies of the hydrolysis of antibiotics catalyzed by a Metallo- $\beta$-lactamase. ABBI$14-569$ 